\title{
The risk of renal disease is increased in lambda myeloma with bone marrow amyloid deposits
}

\author{
Piotr Kozlowski' \\ Scott Montgomery ${ }^{2-4}$ \\ Rahel Befekadu ${ }^{5}$ \\ Victoria Hahn-Strömberg ${ }^{6}$ \\ 'Department of Medicine, School \\ of Medical Sciences, Örebro \\ University, Örebro, Sweden; ${ }^{2}$ Clinical \\ Epidemiology and Biostatistics, \\ School of Medical Sciences, Örebro \\ University, Örebro, Sweden; ${ }^{3}$ Clinical \\ Epidemiology Unit, Karolinska \\ University Hospital, Karolinska \\ Institutet, Stockholm, Sweden; \\ ${ }^{4}$ Department of Epidemiology and \\ Public Health, University College \\ London, London, UK; ${ }^{5}$ Department \\ of Transfusion Medicine, School of \\ Medical Sciences, Örebro University, \\ Örebro, Sweden; ${ }^{6}$ Department \\ of Medical Cell Biology, Uppsala \\ University, Uppsala, Sweden
}

This article was published in the following Dove Press journal: Journal of Blood Medicine 6 March 2017

Number of times this article has been viewed

Background: Light chain amyloidosis (AL) is a rare deposition disease and is present in $10-15 \%$ of patients with myeloma (MM). In contrast to symptomatic AL in MM, presence of bone marrow (BM) amyloid deposits (AD) in MM is not connected to kidney damage. Renal $\mathrm{AD}$ but not BM-AD occur mostly in MM with lambda paraprotein (lambda MM).

Methods: We investigated amyloid presence in BM clots taken at diagnosis in 84 patients with symptomatic MM and compared disease characteristics in MM with kappa paraprotein (kappa $\mathrm{MM}$ )/lambda MM with and without BM-AD.

Results: Lambda MM with BM-AD was compared with kappa MM without BM-AD, kappa MM with BM-AD, and lambda MM without BM-AD: lambda MM with BM-AD patients had a significantly higher mean creatinine level $(4.23 \mathrm{mg} / \mathrm{dL}$ vs $1.69,1.14$, and $1.28 \mathrm{mg} / \mathrm{dL}$, respectively) and a higher proportion presented with severe kidney failure (6/11 [55\%] vs 6/32 [19\%], 1/22 [5\%], and $3 / 19$ [16\%], respectively). Proteinuria was more common in lambda MM with BM-AD patients compared with kappa MM without BM-AD patients (8/11 [73\%] vs 5/32 [16\%], respectively).

Conclusion: Kidney damage was more common in lambda MM with BM-AD indicating presence of renal AD.

Keywords: plasma cells, neoplasms, amyloidosis, renal insufficiency, proteinuria

\section{Introduction}

Light chain amyloidosis (AL) is a rare disease with deposition of amyloid fibrils containing light chains produced by nonproliferative clonal plasma cell (PC), causing mostly renal damage. The disease can also affect other organs such as the heart, and the gastrointestinal tract and peripheral nervous system. ${ }^{1,2}$ The main symptom of kidney damage in AL is proteinuria (73\%), which when excessive ( $>3 \mathrm{~g} / 24 \mathrm{~h}$ or $>2 \mathrm{~g} / \mathrm{L}$ ) gives rise to nephrotic syndrome ( $36 \%$ of AL patients). ${ }^{1}$ Renal function impairment in AL is present in $50 \%$, and severe renal failure, defined as creatinine $>2.0 \mathrm{mg} / \mathrm{dL}$, in $20 \%$ of patients. ${ }^{1}$ Concomitant AL in myeloma (AL-MM) is observed in $10-15 \%$ of patients. ${ }^{3}$ Renal disease in MM, when verified by kidney biopsy, is caused by amyloid deposits (AD) in $21-30 \%$ of patients. ${ }^{4-6}$ Renal function impairment is present in $50 \%$ (severe in $20 \%$ ) of all MM patients at diagnosis. ${ }^{3}$

The standard diagnostic procedure in AL is a tissue biopsy of the affected organ or fat-pad biopsy.

Bone marrow (BM) amyloid deposits (AD) in AL are found in 60-65\% of patients., ${ }^{2,7}$ Presence of BM-AD in MM is reported in $22-40 \%$ of patients ${ }^{8,9}$ and is more common than "true" symptomatic AL-MM. The former has no impact on survival and no connection
Department of Medicine, Örebro University Hospital, Södra Grev Rosengatan, Örebro 70I 85, Sweden

$\mathrm{Tel}+46$ I9 602 I533

Fax +46 196024580

Email piotr.kozlowski@regionorebrolan.se 
with organ damage ${ }^{8,9}$ in contrast with AL-MM where survival is clearly impaired compared with MM without symptomatic AL. ${ }^{10}$ A recent study indicated that BM-AD in MM were present in only $1 \%$ of patients. ${ }^{11}$ In systemic $\mathrm{AL}^{1,2,7}$ and kidney biopsy-proven AL-MM, ${ }^{4}$ a clear dominance of lambda chain restriction (70-80\% of all cases) is observed. This is compared with MM with BM-AD or fat-pad AD where lambda restriction is less common at $42 \%$ or $36 \%$, respectively. ${ }^{8,12}$

Both MM and systemic AL are caused by clonal PC. Their features and proportion in BM though differ in both diseases as abnormal PC and abnormal PC aggregates are common in $\mathrm{MM}^{13}$ but not in AL. ${ }^{7,13}$ The BM PC count in AL is often low, with three quarters of patients having $<10 \%{ }^{7}$ compared with a mean PC count of $50 \%$ in $\mathrm{MM}^{3}$

Previous studies of BM-AD did not evaluate its clinical significance in MM with kappa paraprotein (kappa MM)/ lambda paraprotein (lambda MM) separately. This is why we used BM samples from MM patients taken at diagnosis to identify patients with amyloid presence and then we compared the disease characteristics in kappa/lambda MM with and without BM-AD. We postulated that patients with lambda $\mathrm{MM}$ and $\mathrm{BM}-\mathrm{AD}$ are at higher risk to have kidney damage.

\section{Patients, materials and methods}

All patients with symptomatic MM diagnosed and treated at the Department of Medicine of Örebro University Hospital during 2000-2005 were identified through the hospital register. The treatment was given in accordance with the Swedish national guidelines. Patients under 70 years of age and without significant comorbidity were recommended to undergo autologous stem cell transplantation (ASCT). BM aspirates taken at diagnosis from posterior iliac crest were retrieved. Congo red staining for BM-AD presence and immunocytochemical staining were performed on formalinfixed and paraffin-embedded BM particle clots. Congo red stain was considered as positive when vascular or interstitial deposits with cherry-red appearance (light microscopy) and apple-green birefringence (polarizing microscopy) were present. The biomedical scientist/hematologist assessing BM samples was blinded to the patients' clinical details. PC count, presence of PC atypia and PC aggregates in BM were described at MM diagnosis by a pathologist.

Clinical/laboratory data were collected retrospectively from patients' medical records. Presence of monoclonal protein in serum/urine and kappa/lambda characteristics was assessed by immunoelectrophoresis and immunofixation. Non-Bence Jones (BJ) proteinuria was calculated by subtracting BJ protein from total urinary protein. The date of last follow-up of the survivors was the 5 th of September,
2015. The study was approved by the Regional Ethical Review Board in Uppsala, Sweden, (2011/356), in accordance with the Declaration of Helsinki, and written informed consent was obtained from all of the patients.

\section{Statistics}

Cross-tabulation with the Chi-squared test or means with the two sample $t$-test were used for descriptive statistics. Multiple linear regression was used for outcome variables with continuous distributions. Creatinine levels were logarithmic transformed as they deviated from normal distribution [ $\ln ($ creatinine- 0.3929236$)]$. Binary outcomes were assessed using multiple logistic regression. The analyses of creatinine level and proteinuria were adjusted for age, sex, calcium level and presence of BJ proteinuria $(>0.26 \mathrm{~g} / \mathrm{L})$. The analyses of albumin level were adjusted for uninvolved immunoglobulin level $>4 \mathrm{~g} / \mathrm{L}, \mathrm{M}$ component $<25 \mathrm{~g} / \mathrm{L}$ and presence of light chain MM. The log-rank test was used for overall survival (OS) comparison.

Statistical significance was set as $P$-value $<0.05$ or $95 \%$ confidence interval (CI) that does not include 1.00 (logistic regression) or 0.0 (linear regression). The analyses were performed using SPSS (v.22; IBM).

\section{Results}

A total of 108 patients with symptomatic MM diagnosis were identified. BM particle clots were available in 89 . Three patients with kidney biopsy verified AL, and one patient with nephrotic syndrome and suspected AL was excluded, as well as one patient with nonsecretory MM. The median age in the remaining 84 patients was 70 years (range: 44-93). Beta- 2 microglobulin and BM cytogenetics were assessed in a minority of patients. ASCT was performed in 24 patients. Most of the non-transplanted patients (51/60, 85\%) received an oral melphalan and prednisolone combination (MP) as frontline therapy, two received MP plus thalidomide, three a thalidomide/prednisolone combination and four a vincristine/doxorubicin/dexamethasone combination (VAD regimen). Novel agents (thalidomide, bortezomib, lenalidomide) were used in 40 of 84 (48\%) patients at diagnosis, disease progression or relapse. The patients' characteristics are shown in Table 1.

\section{$B M-A D$ findings and clinical characteristics}

BM-AD was found in 34 of 84 (40\%) patients at diagnosis. Of all clinical/laboratory parameters (listed in Table 1), only presence of hypoalbuminemia (serum albumin $<3.5 \mathrm{~g} / \mathrm{dL}$ ) differed between BM-AD-positive and BM-AD-negative 
Table I Characteristics of 84 MM patients divided into four groups with regard to light chain type and BM-AD presence

\begin{tabular}{|c|c|c|c|c|c|}
\hline & $\begin{array}{l}\text { Total patients, } \\
\text { n (\%) }\end{array}$ & $\begin{array}{l}\text { Lambda, BM-AD } \\
\text { positive, n (\%) }\end{array}$ & $\begin{array}{l}\text { Kappa, BM-AD } \\
\text { negative, } n \text { (\%) }\end{array}$ & $\begin{array}{l}\text { Kappa, BM-AD } \\
\text { positive, } n(\%)\end{array}$ & $\begin{array}{l}\text { Lambda, BM-AD } \\
\text { negative, n (\%) }\end{array}$ \\
\hline Patients per group & 84 & $11(13)$ & $32(38)$ & $22(26)$ & $19(23)$ \\
\hline Age $>70$ years & $41(49)$ & $4(36)$ & $14(44)$ & $13(59)$ & $10(53)$ \\
\hline Males & $4 I(49)$ & $4(36)$ & $15(47)$ & $13(59)$ & $9(47)$ \\
\hline \multicolumn{6}{|l|}{ MM type } \\
\hline $\lg G$ & $50(60)$ & $6(55)$ & $17(53)$ & $16(72)$ & II (58) \\
\hline $\lg A$ & $16(19)$ & $2(18)$ & $10(31)$ & $3(14)$ & I (5) \\
\hline Light chain & $18(21)$ & $3(27)$ & $5(16)$ & $3(14)$ & $7(37)$ \\
\hline \multicolumn{6}{|l|}{ Durie-Salmon stage } \\
\hline I & II (I3) & I (9) & $3(9)$ & $5(23)$ & $2(10)$ \\
\hline ॥ & $35(42)$ & $3(27)$ & $14(44)$ & $8(36)$ & $10(53)$ \\
\hline III & $38(45)$ & $7(64)$ & $15(47)$ & $9(4 I)$ & $7(37)$ \\
\hline Creatinine $>2.0 \mathrm{mg} / \mathrm{dL}$ & $16(19)$ & $6(55)$ & $6(19)^{*}$ & I $(5)^{*}$ & $3(16)^{*}$ \\
\hline Need of dialysis & $8(10)$ & $4(36)$ & $2(6)^{*}$ & I $(5)^{*}$ & I $(5)^{*}$ \\
\hline \multicolumn{6}{|l|}{ Non-BJ proteinuria ${ }^{a}$} \\
\hline$>0.2 \mathrm{~g} / \mathrm{L}^{\mathrm{a}}$ & $32(39)$ & $8(73)$ & $5(16)^{*}$ & $10(45)$ & $9(47)$ \\
\hline$>2 \mathrm{~g} / \mathrm{L}^{\mathrm{a}}$ & $4(5)$ & I (9) & $\mathrm{I}(3)$ & 0 & $2(11)$ \\
\hline $\mathrm{BJ}$ proteinuria $>0.26 \mathrm{~g} / \mathrm{L}^{\mathrm{a}}$ & $42(5 I)$ & $8(73)$ & $19(6 I)$ & $7(32)^{*}$ & $8(42)$ \\
\hline Hypoalbuminemia $<3.5 \mathrm{~g} / \mathrm{dL}$ & $62(74)$ & $6(55)$ & $27(84)$ & $12(55)$ & $17(89)$ \\
\hline Osteolysis & $64(76)$ & $10(91)$ & $26(81)$ & $13(59)$ & $15(79)$ \\
\hline Hypercalcemia (albumin-adjusted & $20(24)$ & $3(27)$ & $7(22)$ & $6(27)$ & $4(21)$ \\
\hline \multicolumn{6}{|l|}{$\mathrm{Ca}>2.55 \mathrm{mmol} / \mathrm{L})$} \\
\hline Hemoglobin $<10.0 \mathrm{~g} / \mathrm{dL}$ & $28(33)$ & $5(45)$ & II (34) & $6(27)$ & $6(32)$ \\
\hline $\mathrm{CRP}>7 \mathrm{mg} / \mathrm{L}$ & $38(45)$ & $4(36)$ & $13(4 \mid)$ & II (50) & $10(53)$ \\
\hline
\end{tabular}

Notes: a 83 patients (anuria at diagnosis in one). *Indicates statistically significant difference as compared with lambda MM with BM-AD. Abbreviations: MM, myeloma; BM-AD, bone marrow amyloid deposits; BJ, Bence Jones; CRP, C-reactive protein.

patients (18/33 [54\%] vs 44/51 [86\%]; odds ratio [OR] 0.19, 95\% CI: $0.07,0.55 ; P=0.002$; and adjusted OR 0.13, 95\% CI: $0.04,0.46 ; P=0.001)$. Mean albumin level was higher in BM-AD-positive patients $(3.29 \mathrm{~g} / \mathrm{dL}$ [standard deviation, SD 0.70] vs $3.01 \mathrm{~g} / \mathrm{dL}$ [SD 0.55]), and when assessed using multiple linear regression, the association was as follows: unadjusted coefficient $-2.83,95 \% \mathrm{CI}:-5.55,-0.11 ; P$ $=0.042 ;$ and adjusted coefficient $-3.22,95 \% \mathrm{CI}:-5.79$, $-0.66 ; P=0.014$. Heart failure was present in five and peripheral neuropathy in only two patients at diagnosis. The latter developed during the treatment in nine patients, all treated with potentially neurotoxic drugs. Alkaline phosphatase and alanine aminotransferase levels were raised (moderately) in only two patients at diagnosis.

\section{$B M-A D$ findings, renal damage, and survival}

Neither presence of BM-AD at diagnosis nor kappa/lambda characteristics were associated with higher creatinine levels. In order to investigate the significance of BM-AD in both light chain types of MM, four groups were constructed (Tables 1 and 2). Renal failure at diagnosis (creatinine $>2.0 \mathrm{mg} / \mathrm{dL}$ ) and need for dialysis (six patients at diagnosis and two at follow-up) were most common in the group with lambda MM and BM-AD presence (Table 1).
Presence of non-BJ proteinuria $(>0.2 \mathrm{~g} / \mathrm{L})$ was more common in lambda MM with BM-AD compared with kappa MM without BM-AD (Table 1). Assessment by multiple logistic regression produced ORs of 13.88 (95\% CI: 2.70, 71.20; $P=0.002)$ before adjustment and $24.22(95 \%$ CI: $3.60,162.82 ; P=0.001)$ after adjustment. Among patients with severe renal failure (16), all six with lambda $\mathrm{MM}$ with BM-AD presented with non-BJ proteinuria compared with five of nine (56\%) remaining patients (one had anuria) $(P=0.09)$. Urine albumin-to-creatinine ratio (UACR) measurement was performed in 59 patients. Four of eight patients $(50 \%)$ with lambda BM-AD-positive lambda MM presented with UACR > 15 compared with three of $23(13 \%)$ with BM-AD-negative kappa MM, producing unadjusted OR using logistic regression of 6.67 (95\% CI: 1.06, 42.06; $P=0.04)$. The proportion of BM-AD-positive kappa MM and BM-AD negative lambda MM was 5/17 (29\%) and 5/11 (45\%), respectively (nonsignificant as compared with BM-AD-positive lambda MM). There was no association between albumin level and presence of proteinuria $(>0.2 \mathrm{~g} / \mathrm{L})$ or between albumin level and UACR $>15$.

Compared with lambda MM with BM-AD, the other three phenotypes were all associated with statistically significant lower creatinine levels, both before and after adjustment for the potential confounding factors (Table 2). In multivariate 
Table 2 Analysis of factors associated with creatinine level ${ }^{a}$ by linear regression in myeloma patients divided into four groups with regard to light chain type and BM-AD presence

\begin{tabular}{|c|c|c|c|c|}
\hline & Patients (n) & $\begin{array}{l}\text { Mean creatinine } \\
\text { (SD) }(\mathrm{mg} / \mathrm{dL})\end{array}$ & $\begin{array}{l}\text { Coefficient }(95 \% \mathrm{Cl}) \text {, } \\
\text { unadjusted } P \text {-value }\end{array}$ & $\begin{array}{l}\text { Coefficient }(95 \% \mathrm{Cl}) \text {, } \\
\text { adjusted } P \text {-value }{ }^{b}\end{array}$ \\
\hline Lambda, BM-AD positive & $\mathrm{II}$ & $4.23(4.38)$ & Ref & \\
\hline Kappa, BM-AD negative & $32^{b}$ & $1.69(2.07)$ & $-1.07(-1.88,-2.53), 0.011$ & $-1.10(-1.80,-0.40), 0.003$ \\
\hline Kappa, BM-AD positive & 22 & $\mathrm{I} .14(0.77)$ & $-1.21(-2.07,-0.35), 0.006$ & $-1.03(-1.80,-0.26), 0.009$ \\
\hline Lambda, BM-AD negative & 19 & $1.28(1.12)$ & $-1.10(-1.98,-0.22), 0.015$ & $-0.90(-1.67,-0.14), 0.022$ \\
\hline \multicolumn{5}{|l|}{ BJ proteinuria } \\
\hline$<0.26 \mathrm{~g} / \mathrm{L}$ & 41 & $\mathrm{I} .00(0.76)$ & Ref & \\
\hline$>0.26 \mathrm{~g} / \mathrm{L}$ & 42 & $2.53(2.99)$ & 0.87 (0.37, I.37), $0.00 \mathrm{I}$ & $0.72(0.26,1.18), 0.003$ \\
\hline Females & 43 & $\mathrm{I} .48(\mathrm{I} .80)$ & Ref & \\
\hline Males & $4 I^{b}$ & $2.10(2.72)$ & $0.60(0.90, I . I I), 0.022$ & $0.50(0.05,0.95), 0.03$ \\
\hline Calcium level (albumin-adjusted) & 84 & $1.79(2.30)$ & $\mathrm{I} .87(0.86,2.87),<0.00 \mathrm{I}$ & $\mathrm{I} .63(0.7 \mathrm{I}, 2.54), 0.00 \mathrm{I}$ \\
\hline Age & 84 & $\mathrm{I} .79(2.30)$ & $0.01(-0.02,-0.03), 0.68$ & $0.01(-0.01,0.03), 0.34$ \\
\hline
\end{tabular}

Notes: a Creatinine levels were log-transformed due to a non-normal distribution. bone patient was omitted in multivariate analysis due to anuria at diagnosis.

Abbreviations: BM-AD, bone marrow amyloid deposits; SD, standard deviation; Cl, confidence interval; Ref, reference; BJ, Bence Jones.

analysis, creatinine level was positively associated with albumin-adjusted calcium level, BJ proteinuria $>0.26 \mathrm{~g} / \mathrm{L}$ and male sex (Table 2). Survival was not associated with presence of BM-AD, and in lambda BM-AD-positive MM, did not differ significantly from OS in the remaining three groups (data not shown).

\section{$B M-A D$ and cytopathological features}

PC atypia was slightly more common in BM-AD-negative patients (48/51 [94\%] vs 27/33 [82\%], $P=0.08$ ]. There was no significant correlation between presence of BM-AD at diagnosis and BM PC count. Proportion of patients with abnormal PC aggregates was not different in AD-positive and AD-negative BM.

\section{Discussion}

We identified AD in BM clots in $40 \%$ of MM patients at diagnosis, which is similar to previous findings in BM smears. ${ }^{8}$ Particle clot preparation is commonly used in Sweden and is sometimes advantageous as proteins and nucleic acids can be damaged during a decalcification procedure of the biopsy. ${ }^{14}$ This may explain the lower frequency of AD in $\mathrm{BM}$ biopsy (25\%) compared with BM aspirate (40\%) in previous studies. ${ }^{8,9}$ There were no differences (except albumin level/ hypoalbuminemia) in laboratory/clinical characteristics of the two groups with and without presence of BM-AD, similar to the results from previous studies. ${ }^{89}$ Despite this, we identified a small but distinctive group of patients with lambda $\mathrm{MM}$ and BM-AD presenting with higher mean creatinine levels, more common kidney failure (nearly $60 \%$ had severe renal failure at diagnosis) and need for dialysis, with more pronounced proteinuria and albuminuria. This indicates that "true" renal amyloidosis was probably more common in this group. Conversely, kidney damage was rare in kappa MM without BM-AD.

There are differences in the pathogenesis of kidney damage in lambda and kappa MM. AL was found to be more common in lambda (80\%) and monoclonal immunoglobulin deposition disease (MIDD) and even MM-cast nephropathy (MCN) in kappa MM among patients undergoing renal biopsy. ${ }^{4-6}$ Twenty-four-hour proteinuria was significantly higher in MM-associated amyloidosis compared with MIDD and MCN. Nephrotic syndrome in MM was caused by amyloidosis in $54-70 \%$ of patients. ${ }^{4,5}$ In our cohort, all patients with BM-AD-positive lambda MM and severe renal failure had proteinuria, in contrast to only half of the remaining patients with severe renal failure, suggesting different causes of renal damage.

Not all AL-MM can be identified using BM Congo red stain, as even in systemic AL, BM-AD was not present in $35-40 \%$ of patients. ${ }^{2,7}$ Interestingly, creatinine levels in systemic $\mathrm{AL}$ with $\mathrm{BM}-\mathrm{AD}$ were higher compared with those without BM-AD. ${ }^{2}$ Even multi-organ involvement was more common in the former group. ${ }^{2}$ Both findings indicate more advanced amyloidosis when BM-AD is present. Renal and cardiac amyloidosis was more common in lambda AL compared with kappa, and the association of different subtypes of light chain variable region genes with different organ tropism was observed previously. ${ }^{15}$

Perhaps surprisingly, albumin levels were lower in BMAD-negative compared with BM-AD-positive patients. This is unlikely to be explained by urinary protein loss, as albumin levels did not differ between patients with presence/absence of proteinuria/albuminuria. Low albumin is recognized as a poor prognostic factor in $\mathrm{MM}^{3}$ and probably indicates more aggressive disease. 
Another difference between BM-AD-positive and BMAD-negative disease, suggesting the involvement of different biological processes, is that PC atypia was slightly less common in the former group. This is consistent with the "normal" appearance of $\mathrm{PC}$ in $\mathrm{AL} .^{7}$ There was no difference in mean $\mathrm{PC}$ count in $\mathrm{BM}$ between $\mathrm{AD}$-positive and $\mathrm{AD}$-negative phenotypes in our cohort, although lower mean PC infiltrate level was demonstrated previously in renal AL-MM compared with other nephropathies in MM. ${ }^{4}$

Similarly to a previous report, ${ }^{8}$ we did not identify any survival disadvantage in patients with BM-AD. AD findings in fat-pad biopsy in MM are considered a poor prognostic factor (even when subclinical) ${ }^{16}$ but not when ASCT is performed. ${ }^{12}$ Thus, the prognostic significance of extra-renal AD remains uncertain. In spite of this, diagnosing symptomatic $\mathrm{AL}$, especially in younger MM patients, is crucial as the treatment-related toxicity and mortality is higher for systemic AL compared with MM in an ASCT setting. ${ }^{17}$ Even tolerability to novel agents such as thalidomide is poorer in systemic AL. ${ }^{18,19}$ The prognosis in AL-MM remains dismal with median survival of only 9.1 months from AL diagnosis. ${ }^{10}$ Renal biopsy is not always possible in frail MM patients especially those with a bleeding tendency. Combining fat and BM biopsy in AL could increase disease detection to $96 \%{ }^{7}$ Simultaneous assessment of kappa/lambda ratio in serum and in urine can indicate renal amyloidosis in MM. ${ }^{6}$

Our study has some potential limitations. The number of lambda MM patients with BM-AD was small. Albuminuria was not measured in all patients. Assessment of $\mathrm{AD}$ in $\mathrm{BM}$ can be difficult as indicated by the discrepancies between studies of AD-BM prevalence in MM. ${ }^{8,9,11}$ Creatinine level is not the most reliable renal function measurement, but this was all that was available for the majority of our patients.

\section{Conclusion}

We confirmed that BM-AD in MM is a relatively common finding, but in contrast with conclusions from other studies, it may have clinical significance. The more prevalent renal disease in lambda MM with BM-AD suggests there could be two separate types of MM-associated BM amyloidosis (possibly even in fat tissue): kappa, predominantly without organ involvement, and lambda signifying "true", systemic amyloidosis mainly causing kidney damage.

The identification of BM amyloid in MM should be assessed together with clinical presentation, light chain type and probably fat-pad biopsy. For a certain diagnosis, biopsy of the affected organ remains the gold standard. Higher albumin levels in patients with BM-AD and slightly less frequent PC atypia suggest that biological processes in $\mathrm{MM}$ with and without BM-AD may differ.

\section{Acknowledgment}

This study was supported by grants from the Research Committee of Region Örebro County Council (Örebro, Sweden).

\section{Disclosure}

The authors report no conflicts of interest in this work.

\section{References}

1. Kyle RA, Gertz MA. Primary systemic amyloidosis: clinical and laboratory features in 474 cases. Semin Hematol. 1995;32(1):45-59.

2. Cowan AJ, Seldin DC, Skinner M, et al. Amyloid deposits in the bone marrow of patients with immunoglobulin light chain amyloidosis do not impact stem cell mobilization or engraftment. Biol Blood Marrow Transplant. 2012;18(12):1935-1938.

3. Kyle RA, Gertz MA, Witzig TE, et al. Review of 1027 patients with newly diagnosed multiple myeloma. Mayo Clin Proc. 2003;78(1):21-33.

4. Nasr SH, Valeri AM, Sethi S, et al. Clinicopathologic correlations in multiple myeloma: a case series of 190 patients with kidney biopsies. Am J Kidney Dis. 2012;59(6):786-794.

5. Montseny JJ, Kleinknecht D, Meyrier A, et al. Long-term outcome according to renal histological lesions in 118 patients with monoclonal gammopathies. Nephrol Dial Transplant. 1998;13(6):1438-1445.

6. Gerth J, Sachse A, Busch M, et al. Screening and differential diagnosis of renal light chain-associated diseases. Kidney Blood Press Res. 2012;35(2):120-128.

7. Swan N, Skinner M, O’Hara CJ. Bone marrow core biopsy specimens in AL (primary) amyloidosis. A morphologic and immunohistochemical study of 100 cases. Am J Clin Pathol. 2003;120(4):610-616.

8. Petruzziello F, Zeppa P, Catalano L, et al. Amyloid in bone marrow smears of patients affected by multiple myeloma. Ann Hematol. 2010; 89(5):469-474.

9. Petruzziello F, Zeppa P, Ciancia G, et al. Cytological and histological detection of amyloid deposits in bone marrow of patients affected by multiple myeloma. Leuk Lymphoma. 2011;52(12):2304-2307.

10. Madan S, Dispenzieri A, Lacy MQ, et al. Clinical features and treatment response of light chain (AL) amyloidosis diagnosed in patients with previous diagnosis of multiple myeloma. Mayo Clin Proc. 2010; 85(3):232-238.

11. Siragusa S, Morice W, Gertz MA, et al. Asymptomatic immunoglobulin light chain amyloidosis (AL) at the time of diagnostic bone marrow biopsy in newly diagnosed patients with multiple myeloma and smoldering myeloma. A series of 144 cases and a review of the literature. Ann Hematol. 2011;90(1):101-106.

12. Desikan KR, Dhodapkar MV, Hough A, et al. Incidence and impact of light chain associated (AL) amyloidosis on the prognosis of patients with multiple myeloma treated with autologous transplantation. Leuk Lymphoma. 1997;27(3-4):315-319.

13. Wei A, Juneja S. Bone marrow immunohistology of plasma cell neoplasms. J Clin Pathol. 2003;56(6):406-411.

14. Lee SH, Erber WN, Porwit A, Tomonaga M, Peterson LC; International Council for Standardization in Hematology. ICSH guidelines for the standardization of bone marrow specimens and reports. Int J Lab Hematol. 2008;30(5):349-364.

15. Abraham RS, Geyer SM, Price-Troska TL, et al. Immunoglobulin light chain variable $(\mathrm{V})$ region genes influence clinical presentation and outcome in light chain-associated amyloidosis (AL). Blood. 2003;101(10): 3801-3808. 
16. Vela-Ojeda J, García-Ruiz Esparza MA, Padilla-González Y, et al. Multiple myeloma-associated amyloidosis is an independent high-risk prognostic factor. Ann Hematol. 2009;88(1):59-66.

17. Bahlis NJ, Lazarus HM. Multiple myeloma-associated AL amyloidosis: is a distinctive therapeutic approach warranted? Bone Marrow Transplant. 2006;38(1):7-15.
18. Palladini G, Perfetti V, Perlini S, . The combination of thalidomide and intermediate-dose dexamethasone is an effective but toxic treatment for patients with primary amyloidosis (AL). Blood. 2005;105(7):2949-2951.

19. Dispenzieri A, Lacy MQ, Rajkumar SV, et al. Poor tolerance to high doses of thalidomide in patients with primary systemic amyloidosis. Amyloid. 2003;10(4):257-261.

\section{Publish your work in this journal}

The Journal of Blood Medicine is an international, peer-reviewed, open access, online journal publishing laboratory, experimental and clinical aspects of all aspect pertaining to blood based medicine including but not limited to: Transfusion Medicine; Blood collection, Donor issues, Transmittable diseases, and Blood banking logistics; Immunohematology; Artificial and alternative
Dovepress

blood based therapeutics; Hematology; Biotechnology/nanotechnology of blood related medicine; Legal aspects of blood medicine; Historical perspectives. The manuscript management system is completely online and includes a very quick and fair peer-review system. Visit http://www.dovepress.com/ testimonials.php to read real quotes from published authors. 